Nota / Note

\title{
TESTES DE ENVELHECIMENTO ACELERADO E DE DETERIORAÇÃO CONTROLADA PARA AVALIAÇÃO DO VIGOR DE SEMENTES DE SOJA
}

\author{
Júlio Marcos Filho ${ }^{1,2 *}$; Ana Dionisia Coelho Novembre ${ }^{1,2}$; Helena Maria Carmignani Pescarin \\ Chamma $^{1}$ \\ ${ }^{1}$ Depto. de Produção Vegetal - USP/ESALQ - C.P. 9 - CEP: 13418-900 - Piracicaba, SP. \\ Bolsista do CNPq. \\ *Autor correspondente <jmarcos@carpa.ciagri.usp.br>
}

RESUMO: A avaliação do de vigor é um importante componente no controle de qualidade da indústria de sementes. A pesquisa, conduzida com sementes de soja [Glycine max (L.) Merrill], não classificadas pelo tamanho, dos cultivares BR-37 e Embrapa 48, teve como objetivos estudar diferentes procedimentos para a distribuição das amostras na superfície da tela metálica das caixas plásticas, que constituem compartimentos individuais (câmaras internas), durante os períodos de exposição ao envelhecimento acelerado e de comparar os resultados com os do teste de deterioração controlada. No teste de envelhecimento acelerado foram utilizadas quatro subamostras para cada lote, previamente pesadas $(42,5 \mathrm{~g})$ ou distribuídas de modo a formar uma camada única de sementes na superfície da tela metálica. A manutenção da umidade relativa no interior de cada caixa plástica foi efetuada de duas maneiras: através da adição de $40 \mathrm{~mL}$ de água (100\% U.R.) ou de $40 \mathrm{~mL}$ de solução saturada de cloreto de sódio (76\% U.R.). As caixas plásticas permaneceram em B.O.D., a $41^{\circ} \mathrm{C}$, durante 48 ou 72 horas. Em seguida, conduziu-se teste de germinação, a $25^{\circ} \mathrm{C}$, com avaliação única aos quatro dias. $\mathrm{O}$ teste de deterioração controlada foi conduzido a $40^{\circ} \mathrm{C}$ durante 48 horas, após o ajuste do grau de umidade das sementes para $15,5 \%$, seguido por germinação durante quatro dias. Os dois sistemas usados para a distribuição das amostras mantiveram a sensibilidade do teste de envelhecimento acelerado para identificar diferenças no vigor dos lotes avaliados, independentemente do uso de água ou de solução salina e do período de envelhecimento. $O$ teste de deterioração controlada constitui alternativa tão consistente quanto o teste de envelhecimento acelerado para a avaliação do vigor de sementes de soja.

Palavras-chave: Glycine max, sementes, análise, potencial fisiológico

\section{ACCELERATED AGING AND CONTROLLED DETERIORATION SEED VIGOR TESTS FOR SOYBEAN}

\begin{abstract}
Seed vigor testing is an important component for the seed industry quality control. This study was conducted to compare different procedures of the accelerated aging and the controlled deterioration tests to evaluate soybean [Glycine max (L.) Merrill] seed vigor. Two soybean cultivars, BR-37 and Embrapa 48, each represented by different seed lots were used. All lots were submitted to two procedures of placing seed samples on the accelerated aging tray: 1 . the addition of a monolayer of seeds or 2 . the use of a standard weight $(42.5 \mathrm{~g})$ of seeds. The study also examined the possibility of using a saturated $\mathrm{NaCl}$ solution in substitution to water in comparison to the standard accelerated aging test $\left(48\right.$ and $72 \mathrm{~h}$ at $41^{\circ} \mathrm{C}$, followed by germination during four days). Controlled deterioration test was conducted using seeds with water content raised to $15.5 \%$, placed in laminated aluminum bags and maintained in water-bath at $40^{\circ} \mathrm{C}$ for $48 \mathrm{~h}$ followed by a germination test. Information provided by the accelerated aging test was not influenced significantly by the procedure used to place seed samples on the tray, both in the standard test and in the salt accelerated aging test. This procedure and also the controlled deterioration test may be considered as efficient alternatives for the assessment of soybean seed vigor allowing for results as consistent as those provided by the standard accelerated aging test. Key words: Glycine max, seed analysis, physiological quality
\end{abstract}

\section{INTRODUÇÃO}

O teste de envelhecimento acelerado é reconhecido como um dos mais populares para a avaliação do vigor de sementes de várias espécies, sendo capaz de proporcionar informações com alto grau de consistência (TeKrony, 1995).

A metodologia, descrita por Delouche \& Baskin
(1973), passou a ser estudada com maior profundidade, tendo sido geradas importantes contribuições para a padronização do teste (Baskin, 1977; McDonald \& Phaneendranath, 1978; Tao, 1979; Baskin, 1981; TeKrony, 1985; Tomes et al., 1988). No entanto, ainda persistem dúvidas quanto a aspectos específicos da metodologia e outros que podem ser aprimorados. 
O procedimento recomendado para a condução do teste, com sementes de soja, especifica o uso de amostras de $40 \mathrm{~g}$ (sementes "pequenas") a $45 \mathrm{~g}$ (sementes "grandes"), distribuídas sobre superfície da tela metálica colocada no interior da caixa plástica (câmara interna) utilizada para o teste (Tomes et al., 1988). Essa especificação tem como objetivo assegurar que uma camada única de sementes seja distribuída sobre a tela metálica, de modo a garantir a exposição uniforme das amostras às condições de temperatura e umidade relativa estabelecidas para o teste, para atenuar ou eliminar os possíveis efeitos do tamanho das sementes que constituem a amostras avaliadas. No entanto, não há caracterização do que seriam as sementes "pequenas" ou "grandes"; acrescente-se que a prática demonstra a possibilidade da constituição de camadas sobrepostas de sementes sobre a superfície da tela, dependendo do tamanho das sementes que constituem as amostras e do peso utilizado. Consequentemente, os objetivos dessa recomendação podem não ser completamente atingidos, conforme demonstraram Fahim \& McDonald (s.d.), em estudo preliminar sobre $o$ assunto.

Outro teste baseado na resistência das sementes ao estresse provocado pela exposição à temperatura $\mathrm{e}$ umidade relativa elevadas, é o da deterioração controlada, recomendado principalmente para hortaliças (Powell \& Matthews, 1981). Nesse caso, o teor de água de todas as amostras de sementes é ajustado para valor de, no mínimo, 15,5\%, previamente à instalação do teste. Assim, o efeito do teor de água sobre a deterioração é direto, ao contrário do que se verifica no teste de envelhecimento acelerado.

As sementes umedecidas são mantidas em banho-maria, a $40^{\circ} \mathrm{C}$, durante 48 horas e, em seguida, as amostras são colocadas para germinar. A percentagem de plântulas normais é proporcional ao vigor das sementes. Em estudo inicial, Rossetto \& Marcos Filho (1995) constaram que a deterioração controlada foi mais sensível que o envelhecimento acelerado para detectar diferenças de vigor entre lotes de sementes de soja. Portanto, apesar da reconhecida eficiência do teste de envelhecimento acelerado, a intensificação dos estudos referentes à deterioração controlada constitui alternativa interessante para inclusão em programas de controle de qualidade. Embora relativamente pouco estudado para a avaliação do vigor de sementes de soja, trata-se de um teste relativamente simples, que não exige conhecimentos sofisticados, investimentos significativos e não apresenta dificuldades acentuadas para padronização.

Assim, a presente pesquisa teve como objetivos: a) comparar a eficiência do uso de amostras com peso pré-determinado e a distribuição de camada simples de sementes sobre a superfície da tela metálica, para a condução do teste de envelhecimento acelerado; b) comparar a sensibilidade dos testes de envelhecimento acelerado e de deterioração controlada para avaliação do vigor de sementes de soja.

\section{MATERIAL E MÉTODOS}

A pesquisa foi conduzida com sementes de dois cultivares de soja, BR-37 (nove lotes) e Embrapa 48 (sete lotes), não classificadas pelo tamanho e com potenciais fisiológicos distintos. A utilização de cultivares diferentes é importante porque a resposta das sementes a estresses, como as provocadas pelas condições dos testes de envelhecimento acelerado e de deterioração controlada, pode ser afetada significativamente pelo genótipo; esta situação deve ser avaliada em pesquisas sobre o assunto. Por outro lado, a inclusão de lotes de germinação compatível com os padrões de comercialização de sementes, mas com potenciais fisiológicos distintos, é fundamental para a identificação do poder discriminatório ou sensibilidade dos testes à amplitude de variação do vigor das amostras de sementes avaliadas.

Foram utilizadas sementes recém-colhidas, conduzindo-se os testes de envelhecimento acelerado e deterioração controlada, de acordo com os seguintes procedimentos:

Envelhecimento acelerado: foram utilizadas caixas plásticas $(11 \times 11 \times 3 \mathrm{~cm})$ como compartimento individual. A umidade relativa no interior dessas caixas foi obtida pela adição de $40 \mathrm{~mL}$ de água (para obtenção de, aproximadamente, $100 \%$ U.R.) ou de $40 \mathrm{~mL}$ de solução saturada de cloreto de sódio ( $76 \%$ U.R.) ao fundo de cada caixa plástica, conforme Jianhua \& McDonald (1996) e Panobianco \& Marcos Filho (1998).

As amostras foram distribuídas de duas maneiras, na superfície da tela metálica mantida no interior de cada caixa plástica: na primeira, as sementes constituíram uma camada única, tomando toda a superfície da tela metálica, independentemente do número e do peso das sementes; na segunda, a superfície da tela foi preenchida com amostras de 42,5 $\mathrm{g}$, para cada tratamento (nesta situação, há certa superposição das sementes distribuídas sobre a tela).

Os períodos de permanência das amostras no interior da câmara, a $41^{\circ} \mathrm{C}$, foram de 48 e de 72 horas, seguido-se a condução de teste de germinação durante quatro dias, em rolos de papel-toalha, a $25^{\circ} \mathrm{C}$, computando-se as percentagens de plântulas normais para cada uma das repetições de cada tratamento.

Deterioração controlada: amostras de 250 sementes, para cada tratamento/lote e cultivar, foram umedecidas artificialmente, até atingir teor de água de 15,5\%, conforme Rossetto \& Marcos Filho (1995), e colocadas em saquinhos de alumínio revestidos com plástico, hermeticamente fechados. Estes foram mantidos em banho-maria, a $40^{\circ} \mathrm{C}$, durante 48 horas. Em seguida, foi conduzido teste de germinação, interpretado no quarto dia após a semeadura, conforme o item anterior.

Grau de umidade: avaliado antes e após cada período de envelhecimento acelerado e de deterioração 
controlada, para verificação da uniformidade das condições utilizadas para ambos os testes; a determinação desse parâmetro seguiu as especificações das Regras para Análise de Sementes (Brasil, 1992). O grau de umidade das sementes, no início dos testes, variou de $9,0 \%$ a $10 \%$, para ambos os cultivares.

Procedimento estatístico: as análises dos dados referentes aos testes conduzidos, com exceção para a determinação do grau de umidade, foram efetuados separadamente para cada teste e cultivar, estudando-se os efeitos de lotes, com os tratamentos distribuídos em blocos ao acaso. No estudo relativo ao teste de envelhecimento acelerado, esquemas independentes incluíram os efeitos do tipo de distribuição das amostras sobre a tela metálica ou da umidade relativa (água e solução salina) no interior do compartimento individual. As médias foram comparadas pelo método de Tukey $(\mathrm{P}<0,05)$.

\section{RESULTADOS E DISCUSSÃO}

A avaliação do potencial fisiológico de sementes de soja tem merecido especial atenção de vários pesquisadores, refletindo a preocupação causada pelas dificuldades encontradas para a obtenção dos desempenhos desejados com os lotes de sementes comercializados. Dentre os testes disponíveis para a avaliação do vigor das sementes, os de envelhecimento acelerado e de deterioração controlada, estudados na presente pesquisa, têm sido destacados como dos mais eficientes (Rossetto \& Marcos Filho, 1995). Esses testes preenchem os critérios relacionados por Powell \& Matthews (1981) para que um teste de vigor seja considerado consistente.

A germinação de seis dos nove lotes de sementes do cv. BR-37, utilizados neste trabalho, foi superior a $90 \%$ e, portanto, superior à mínima estabelecida para a comercialização de sementes de soja. Os lotes 1, 3 e 4 apresentaram, respectivamente, $79 \%, 71 \%$ e $77 \%$ de germinação.

Nos testes de envelhecimento acelerado conduzidos com as sementes desse cultivar, foi detectado o potencial fisiológico significativamente inferior dos lotes 3 e 4, em relação aos lotes 5, 6, 7 e 8, enquanto os lotes 1, 2 e 9 situaram-se em posição intermediária (Figuras 1 a 4). Essa caracterização mostrou-se independente da umidade relativa do ar no interior dos compartimentos individuais ( $100 \%$ ou $76 \%$ ), do sistema de distribuição das amostras (camada simples ou massa uniforme) e do período de envelhecimento (48 ou 72 horas).

Um dos aspectos controvertidos na condução do teste de envelhecimento acelerado é o procedimento adotado para a distribuição das amostras na superfície da tela metálica colocada no interior de cada compartimento individual (câmara interna). Os primeiros pesquisadores a estudar e a desenvolver esse processo, recomendavam a distribuição de uma camada simples de sementes na superfície da tela, para que todas as sementes pudessem ser expostas, de maneira uniforme, às condições de temperatura e de umidade relativa do ar, características do teste (McDonald \& Phaneendranath, 1978; Tao, 1979). Porém, visando atenuar os possíveis efeitos do tamanho das sementes sobre o seu comportamento no teste de envelhecimento acelerado, Tomes et al. (1988) propuseram a utilização de amostras com massa uniforme (40 a $45 \mathrm{~g}$, dependendo do tamanho da semente), visando a distribuição das sementes de modo a formar camada simples na superfície da tela.

No entanto, a prática tem demonstrado que, dependendo do tamanho das sementes, há superposição entre elas e, portanto, não há formação de camada simples; esse fato também ocorreu no presente trabalho. Há, também, nessa recomendação, indefinição quanto ao que seriam sementes "grandes", "médias" e "pequenas". Essas situações prejudicam a padronização do teste (McDonald \& Phaneendranath, 1978). Por outro lado, de acordo com Marcos Filho et al. (2000), os dois sistemas de distribuição das amostras não atenuaram nem eliminaram os efeitos do tamanho sobre 0 comportamento das sementes no teste.

As percentagens de germinação após o envelhecimento acelerado, nos nove lotes de 'BR-37', foram semelhantes, com raras exceções, quando se compararam os dois sistemas de distribuição das amostras (Figuras 1 a 4 ). Como as informações fornecidas pelo teste, quanto à identificação de diferenças entre o potencial fisiológico dos lotes de sementes avaliados, mostraram praticamente a mesma sensibilidade, os resultados aqui obtidos demonstraram que esses dois sistemas de distribuição das amostras podem ser utilizados para a condução do teste de envelhecimento acelerado. As diferenças no grau de umidade após o envelhecimento não superaram 0,7\%, sob $100 \%$ de umidade relativa do ar, tanto para o período de 48 horas $(25,6 \%$ versus $26,3 \%)$ como para 72 horas $(28,7 \%$ versus $29,2 \%)$. Quando se utilizou a solução salina, essa diferença entre as amostras distribuídas em camada simples e as pré-pesadas foi, no máximo, de $0,2 \%$. Esses valores situam-se dentro da diferença máxima de 2 a $3 \%$ de água entre as amostras (no final do envelhecimento acelerado), considerada segura para a interpretação dos resultados (Tomes et al., 1988).

O uso de solução salina e a conseqüente redução da umidade relativa do ar para $76 \%$, no interior de cada compartimento individual, contribuiu, de maneira acentuada, para que os efeitos do envelhecimento acelerado se tornassem menos drásticos (Figuras 2 e 4). As sementes captaram quantidades significativamente menores de água, atingindo graus de umidade entre $12,2 \%$ e $12,4 \%$, nos dois períodos de envelhecimento, mas o teste não se tornou menos eficiente na detecção de diferenças entre os potenciais fisiológicos dos lotes, 
confirmando as conclusões de Jianhua \& McDonald (1996) e de Panobianco \& Marcos Filho (1998), para sementes de floríferas e de hortaliças, respectivamente. No presente trabalho, notou-se que 0 período de 72 horas mostrou-se mais adequado, quando foi utilizada a solução salina, porque, embora mais drástico, permitiu identificar diferenças mais definidas entre o potencial fisiológico dos lotes, sem a alternância habitualmente verificada em lotes de qualidade intermediária.

Os resultados do teste de deterioração controlada foram equivalentes aos do envelhecimento acelerado, ou seja, os lotes 5, 6, 7 e 8 foram identificados como os mais vigorosos, enquanto os lotes 2, 3 e 4 apresentaram os potenciais fisiológicos mais baixos (Figuras 1 a 4).

De um modo geral, a deterioração controlada promoveu efeitos de intensidade intermediária, em relação aos períodos de 48 e 72 horas de

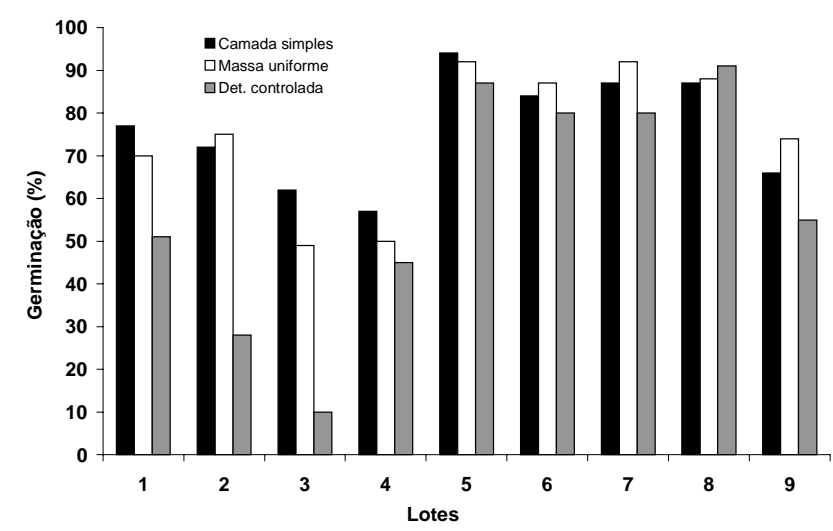

Figura 1 - Sementes de soja, cv. BR-37. Resultados dos testes de deterioração controlada e de envelhecimento acelerado (48horas, $100 \%$ de umidade relativa do ar e dois sistemas de distribuição das amostras).Piracicaba, 1998/99.

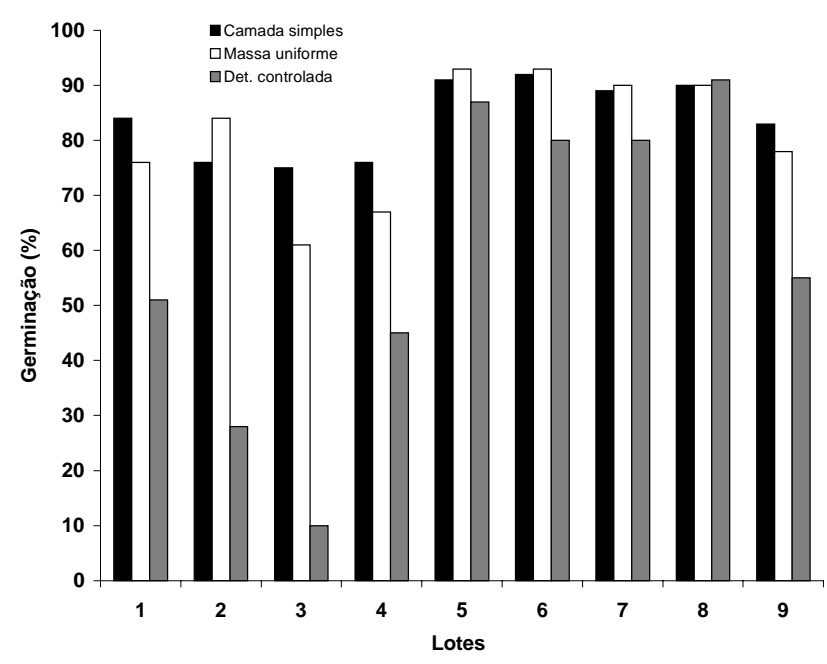

Figura 2 - Sementes de soja, cv. BR-37. Resultados dos testes de deterioração controlada e de envelhecimento acelerado (48 horas, uso de solução salina e dois sistemas de distribuição das amostras). Piracicaba, 1998/99. envelhecimento acelerado, quando este teste foi conduzido sob umidade relativa de $100 \%$; isto ocorreu principalmente quando foram avaliados os lotes de potencial fisiológico inferior. Em outras palavras, a germinação desses lotes (1 a 4) após 48 horas de envelhecimento foi superior à observada após a deterioração controlada; esta, por sua vez, superou a germinação observada após 72 horas de envelhecimento acelerado.

Os efeitos do envelhecimento acelerado, sob umidade relativa de $100 \%$, foram mais drásticos que os desse teste conduzido com o uso de solução salina. De qualquer maneira, o teste de deterioração controlada pode ser considerado um teste eficiente e comparável ao envelhecimento acelerado, confirmando as observações efetuadas por Rossetto \& Marcos Filho (1995), pois conseguiu discriminar o potencial fisiológico dos lotes, com a mesma eficiência verificada para o teste de envelhecimento acelerado.

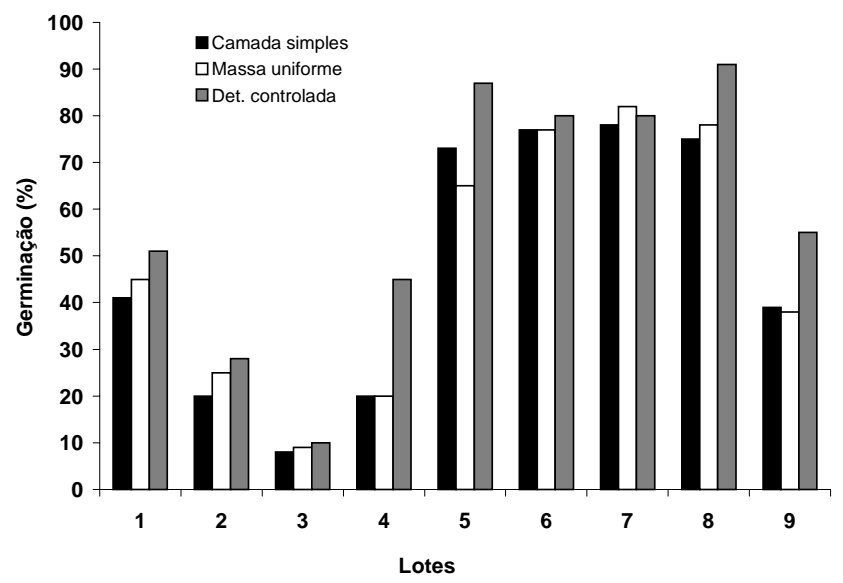

Figura 3 - Sementes de soja, cv. BR-37. Resultados dos testes de deterioração controlada e de envelhecimento acelerado (72 horas, 100\% de umidade relativa do ar e dois sistemas de distribuição das amostras). Piracicaba, 1998/99.

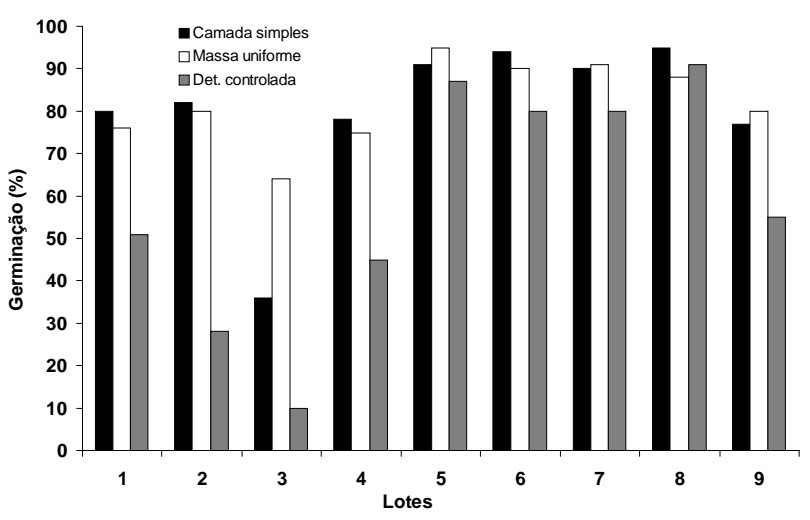

Figura 4 - Sementes de soja, cv. BR-37. Resultados dos testes de deterioração controlada e de envelhecimento acelerado (72 horas, uso de solução salina e dois sistemas de distribuição das amostras). Piracicaba,1998/99. 
Os resultados para os lotes de sementes do cultivar 'Embrapa 48', que possuíam germinação inicial de $83 \%$ a $95 \%$, foram condizentes com os verificados para 'BR-37'. Assim, os testes de envelhecimento acelerado detectaram a superioridade dos lotes $4 a, 5 a$, $6 a$ e $7 a$, em relação aos lotes $1 \mathrm{a}, 2 \mathrm{a}$ e $3 \mathrm{a}$, independentemente do procedimento adotado para a distribuição das amostras, período de envelhecimento e umidade relativa do ar no interior de cada compartimento individual. Desta forma, não foram identificados lotes com qualidade intermediária (Figuras 5 a 8).

As diferenças entre os graus de umidade das sementes distribuídas no sistema de camada simples, em relação às pré-pesadas, foram ainda mais estreitas que as verificadas para o cultivar BR-37, variando de $0,3 \%$ a $0,7 \%$, dependendo do período de envelhecimento sob $100 \%$ de umidade relativa do ar, e de $0,2 \%$, com 0

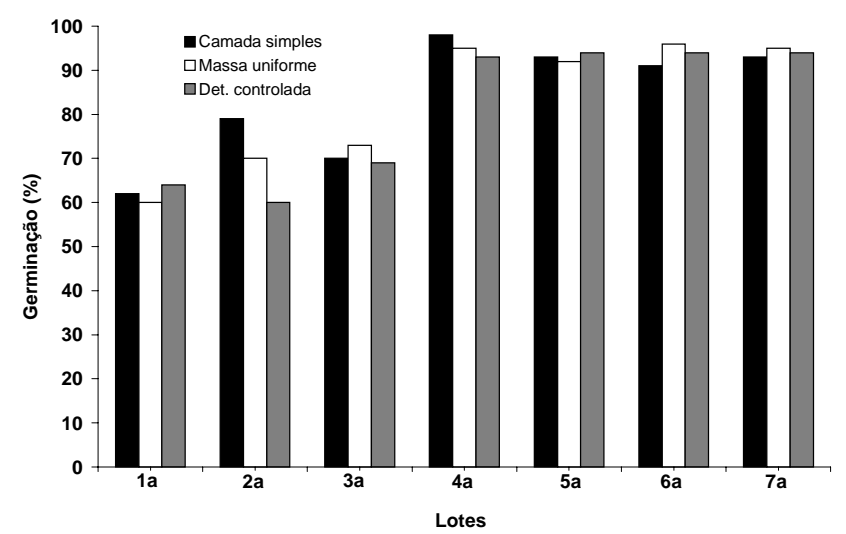

Figura 5 - Sementes de soja, cv. Embrapa 48. Resultados dos testes de deterioração controlada e de envelhecimento acelerado (48 horas, $100 \%$ de umidade relativa do ar e dois sistemas de distribuição das amostras). Piracicaba, 1998/99.

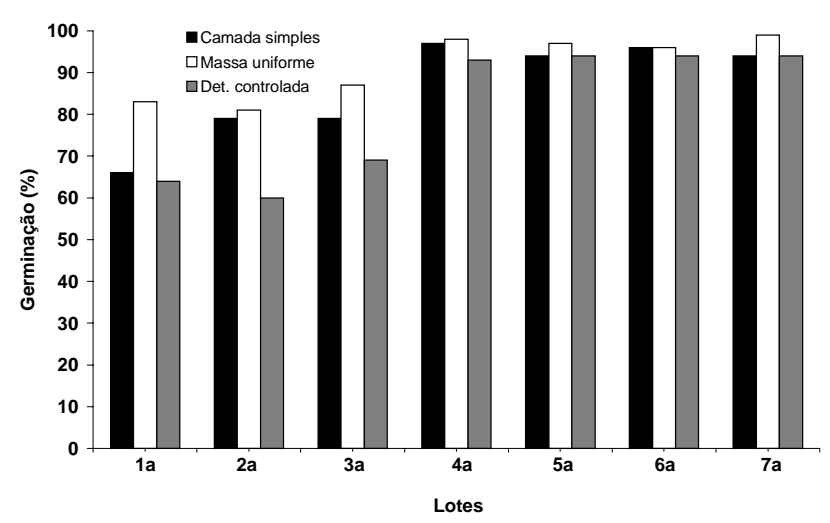

Figura 6 - Sementes de soja, cv. Embrapa 48. Resultados dos testes de deterioração controlada e de envelhecimento acelerado (48 horas, uso de solução salina e dois sistemas de distribuição das amostras). Piracicaba, 1998/99. uso de solução salina. Essas condições mais uniformes, verificadas durante as conduções dos testes com sementes de 'Embrapa 48', permitem explicar as menores variações da percentagem de germinação após o envelhecimento, observadas, de um modo geral, quando se comparam os resultados provenientes dos dois métodos de distribuição das amostras.

Da mesma forma, o comportamento das sementes no teste de deterioração controlada permite ressaltar os comentários efetuados durante a exposição dos resultados obtidos para as sementes de 'BR-37', quanto à detecção de diferenças no potencial fisiológico dos lotes avaliados, aos efeitos comparativos com os dois períodos de envelhecimento e à eficiência em relação ao teste de envelhecimento acelerado.

Assim, os resultados obtidos no presente trabalho não identificaram efeitos da distribuição das amostras na superfície da tela metálica colocada no

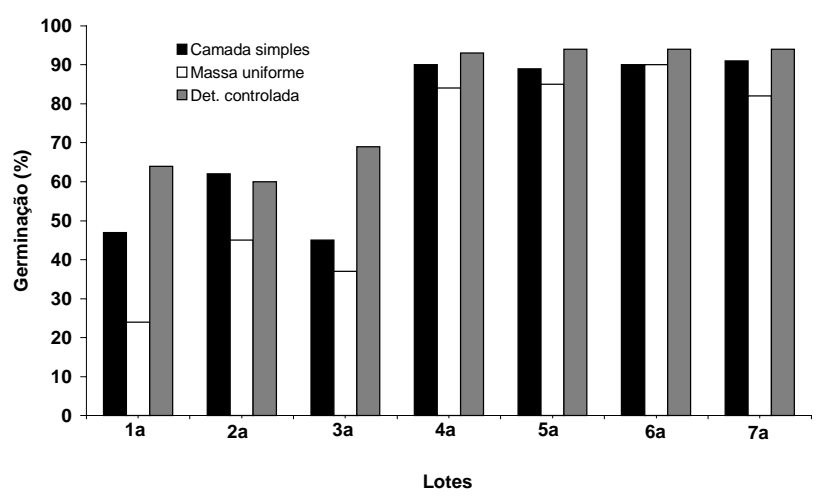

Figura 7 - Sementes de soja, cv. Embrapa 48. Resultados dos testes de deterioração controlada e de envelhecimento celerado (72 horas, 100\% de umidade relativa do ar e dois sistemas de distribuição das amostras). Piracicaba, 1998/99.

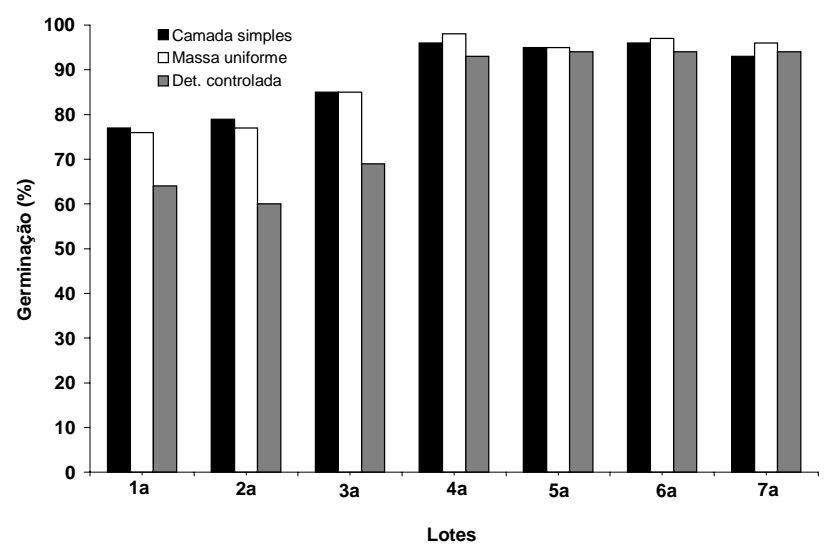

Figura 8 - Sementes de soja, cv. Embrapa 48. Resultados dos testes de deterioração controlada e de envelhecimento acelerado (72 horas, uso de solução salina e dois sistemas de distribuição das amostras). Piracicaba,1998/99. 
interior dos compartimentos individuais (câmaras internas), demonstrando a semelhança das informações obtidas. Diante dessa consideração, sugere-se a distribuição das amostras em camada simples, evitandose a operação adicional da pesagem e simplificando o procedimento.

Confirmou-se a existência de novas alternativas para a avaliação do vigor de sementes de soja, representadas pelo teste de envelhecimento acelerado, com utilização de solução salina, e pela possibilidade de inclusão do teste de deterioração controlada como componente de programas de controle de qualidade.

\section{CONCLUSÕES}

A distribuição das amostras formando camada simples na superfície da tela metálica de cada compartimento individual ou a utilização de amostras com massa uniforme $(42,5 \mathrm{~g})$ não modifica a capacidade do teste de envelhecimento acelerado identificar diferenças no potencial fisiológico de lotes de sementes de soja.

O teste de deterioração controlada constitui uma alternativa tão consistente quanto o teste de envelhecimento acelerado para a avaliação do vigor de sementes de soja.

\section{REFERÊNCIAS BIBLIOGRÁFICAS}

BASKIN, C.C. Vigor testing methods: accelerated aging. Association of Official Seed Analysts Newsletter, v.51, p.42-52, 1977.

BASKIN, C.C. Accelerated aging test. In: PERRY, D.A. (Ed.) Handbook fo vigour test methods. Zurich: International Seed Testing Association, 1981. p.43-48.

BRASIL. Ministério da Agricultura. Regras para análise de sementes. Brasília: SNAD/DNPV/CLAV, 1992. 365 p.

DELOUCHE, J.C.; BASKIN, C.C. Accelerated aging techniques for predicting the relative storability of seed lots. Seed Science and Technology, v.1, p.427-452, 1973.
FAHIM, E.S.; McDONALD, M.B. The effect of seed size on the accelerated aging vigor test for soybean. Columbus: Ohio State University, Dept. of Horticulture and Crop Science, s.d.

JIANHUA, Z.; McDONALD, M.B. The salt accelerated aging test for small-seeded crops. Seed Science and Technology, v.25, p.123-131, 1996.

MARCOS FILHO, J.; NOVEMBRE, A. D.C.; CHAMMA, H.M.C.P. Tamanho da semente e o teste de envelhecimento acelerado para soja. Scientia Agricola, v.57, p.473-482, 2000.

McDONALD, M.B.; PHANEENDRANATH, B.R. A modified accelerated aging vigor test procedure. Journal of Seed Technology, v.3, p.27-37, 1978.

PANOBIANCO, M.; MARCOS FILHO, J. Comparação entre métodos para avaliação da qualidade fisiológica de sementes de pimentão. Revista Brasileira de Sementes, v.20, p.306310, 1998

POWELL, A.A.; MATTHEWS, S. Evaluation of controlled deterioration, a new vigour test for crop seeds. Seed Science and Technology, v.9, p.633-640, 1981.

ROSSETTO, C.A.V.; MARCOS FILHO, J. Comparação entre os métodos de envelhecimento acelerado e de deterioração controlada para avaliação da qualidade fisiológica de sementes de soja. Scientia Agricola, v.52, p.123-131, 1995.

TAO, K.L. An evaluation of alternative methods of accelerated aging seed test for soybeans. Journal of Seed Technology, v.3, p.30-40, 1979.

TeKRONY, D.M. An evaluation of the accelerated aging test for soybeans. Association of Official Seed Analysts Newsletter, v.59, p.86-96, 1985.

TeKRONY, D.M. Accelerated aging test. In: HAMPTON, J.G.; TeKRONY, D.M. (Ed.) Handbook of vigour test methods. 3.ed. Zurich: International Seed Testing Association, 1995. p.35-50.

TOMES, L.J.; TeKRONY, D.M.; EGLI, D.B. Factors influencing the tray accelerated aging test for soybean seed. Journal of Seed Technology, v.12, p.24-35, 1988.

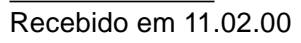

